\title{
Management Difficulties of Patients Suffering from Dementia during Covid-19 Pandemic: Bangladesh Perspective
}

\author{
Maliha Hakim \\ Professor \& Head, Department of Neurology, National Institute of Neurosciences \& Hospital, Dhaka, \\ Bangladesh; Email: drmalihahakim@yahoo.com; Cell no.: +8801309923067
}

Dementia is a disease of old age and is likely to be associated with a variety of comorbidities, especially frailty, which makes this group of patients vulnerable to infection ${ }^{1}$. In addition, they tend to live in a crowded environment such as care homes and needing continuous contact with careers which may further increase their risk of infection. The world is facing a global epidemic of both ageing and dementia. By the year 2050 , the total number of people aged $\geq 60$ years are expected to reach 2 billion from 900 million in 2015; furthermore, the fastest growing group is those above the age of 80 years who are expected to reach 434 million in $2050^{2}$. The global ageing, which has been started initially in high income countries, is now affecting all countries, and almost $80.0 \%$ of the older people are predicting to reside in the low and middle-income countries by the year 2050; however, dementia is also emerging as a global pandemic in older people with more than 50 million people currently living with dementia and the incidence increases by one new case every 3 seconds ${ }^{3}$.

Dementia has been shown to be associated with a significant increased risk of mortality in patients with COVID-19 infection ${ }^{4}$. People with dementia are at high risk of SARS-CoV-2 infection because cognitive symptoms cause difficulty with following safeguarding procedures and living arrangements in care homes facilitate viral spread ${ }^{5}$. Once infected, older adults with dementia are more likely to experience severe virus-related outcomes, including death, than are people without dementia. A homozygous APOE $\varepsilon 4$ genotype is associated with an increased risk of hospitalization for COVID-19, possibly owing to exacerbated inflammation and cytokine production that leads to a cytokine storm ${ }^{6}$. Older adults with dementia, especially those in care homes, are at high risk of worsening psychiatric symptoms and severe behavioral disturbances as a result of social isolation during the pandemic.

The COVID-19 pandemic has had a unique impact on people with Alzheimer disease (AD) as well ${ }^{3}$. As research into this impact has accumulated throughout 2020, a clear picture has emerged that this population is particularly susceptible not just to SARS-CoV-2 infection and its effects, but also to the negative effects of the measures taken worldwide to control the spread of the virus. The restrictions that have been implemented in many countries to control the pandemic have also had important neuropsychiatric consequences for patients with dementia5. In the population as a whole, forced social isolation has led to an increase in reported psychiatric symptoms like stress, anxiety and depression for all individuals; this relationship seems to be moderated by the loneliness associated with prolonged periods of lockdown4. In nursing and care homes, older adults are likely to experience additional distress owing to the absence of relatives who would normally visit them, as well as strict limitations on social activities and interactions with fellow residents. Data collected during the first half of 2020 show that such social isolation during the pandemic is associated with manifestation and/or exacerbation of neuropsychiatric symptoms even in cognitively healthy older adults 5 .

Normally, people living with dementia are the most vulnerable and dependent group in the society. They are relying on the care delivered by family members or professional careers for their day to day survival. During the COVID-19 pandemic, their vulnerability is likely to be exaggerated due to the direct impact of the disease on them and the indirect effects of the pandemic on the social support and the health care system ${ }^{6}$. Older people with dementia are likely to have little information or understanding of the COVID-19 pandemic. Health care safeguarding procedures such as social distancing, hand washing, wearing masks or reporting early symptoms of the infection may be difficult tasks to comprehend in this population exposing them to a higher risk of infection. With increasing social isolation, people with dementia and 
living alone may have less career input which may aggravate their isolation, increasing their loneliness and risk of depression and anxiety. More ever, patients with severe dementia and behavioral problems such as agitation or wandering will undermine every effort to maintain isolation and can be a risk for other individuals especially their careers. The outcome of COVID-19 is likely to be worse when dementia is present. Older people with dementia are likely to have high prevalence of comorbid conditions such as cardiovascular disease, diabetes and increased risk of pneumonia compared to individuals without dementia? These comorbidities have been associated with adverse outcomes and increased mortality in patients with COVID-19. Pneumonia was common in COVID-19 affecting up to $90.0 \%$ of cases in China ${ }^{8}$. In general, the mortality risk due to pneumonia in people with dementia has been reported to be twice that in people without dementia.

Research is ongoing to develop effective anti-COVID-19 agents as well as protective vaccination. Older people with comorbid dementia and frailty have compromised immunity that needs to be considered in current vaccination research to guarantee good immune response and efficacy in this vulnerable population ${ }^{7}$. With global ageing, the number and proportion of older people with dementia will continue to increase, especially in the low-and-middle income countries which have limited resources to face any further pandemic with an increased risk of uncontrollable global spread. Therefore, WHO and world-wide policy makers must take this into account in their future planning for health care. It is a global task to reduce frailty and comorbidity and to promote healthy ageing through adoption of a healthy life style and a widespread access to preventative health care across the world especially in the deprived societies ${ }^{8}$.
New ways of remote communication with people living with dementia still require future investigations. Additionally, care home populations, which tend to have high prevalence of dementia, are likely to continue to increase, and these crowded settings are a risk factor for the spread of infection; therefore, review of current policies and novel regulations are required.

[Journal of National Institute of Neurosciences Bangladesh, 2021;7(2): 90-91]

\section{References}

1. Hariyanto TI, Putri C, Situmeang RF, Kurniawan A. Dementia is a predictor for mortality outcome from coronavirus disease 2019 (COVID-19) infection. European Archives of Psychiatry and Clinical Neuroscience. 2021 Mar;271(2):393-5

2. Mok VC, Pendlebury S, Wong A, Alladi S, Au L, Bath PM, Biessels GJ, Chen C, Cordonnier C, Dichgans M, Dominguez J. Tackling challenges in care of Alzheimer's disease and other dementias amid the COVID-19 pandemic, now and in the future. Alzheimer's \& Dementia. 2020 Nov;16(11):1571-81

3. Brown EE, Kumar S, Rajji TK, Pollock BG, Mulsant BH. Anticipating and mitigating the impact of the COVID-19 pandemic on Alzheimer's disease and related dementias. The American Journal of Geriatric Psychiatry. 2020 Jul 1;28(7):712-21

4. Azarpazhooh MR, Amiri A, Morovatdar N, Steinwender S, Ardani AR, Yassi N, Biller J, Stranges S, Belasi MT, Neya SK, Khorram B. Correlations between COVID-19 and burden of dementia: An ecological study and review of literature. Journal of the neurological sciences. 2020 Sep 15;416:117013

5. Zuin M, Guasti P, Roncon L, Cervellati C, Zuliani G. Dementia and the risk of death in elderly patients with COVID-19 infection: Systematic review and meta-analysis. International Journal of Geriatric Psychiatry. 2021 May;36(5):697-703

6. Wang Q, Davis PB, Gurney ME, Xu R. COVID-19 and dementia: Analyses of risk, disparity, and outcomes from electronic health records in the US. Alzheimer's \& Dementia. 2021 Feb 9.

7. Simonetti A, Pais C, Jones M, Cipriani MC, Janiri D, Monti L, Landi F, Bernabei R, Liperoti R, Sani G. Neuropsychiatric symptoms in elderly with dementia during COVID-19 pandemic: definition, treatment, and future directions. Frontiers in psychiatry. 2020;11

8. Tousi B. Dementia care in the time of COVID-19 pandemic. Journal of Alzheimer's Disease. 2020 Jan 1(Preprint):1-5 